\title{
Efficacy and Safety of Luseogliflozin in Patients with Type 2 Diabetes Complicated by Hepatic Dysfunction: A Single-Site, Single-Arm, Open-Label, Exploratory Trial
}

Hiroaki Seino (D)

Received: December 23, 2020 / Accepted: January 27, 2021 / Published online: February 16, 2021

(C) The Author(s) 2021

\begin{abstract}
Introduction: Improvements in glycemic control and hepatic function are clinically important goals in the treatment of patients with type 2 diabetes mellitus (T2DM) complicated by hepatic dysfunction. The favorable effects of the sodium-glucose co-transporter inhibitor luseogliflozin on hepatic dysfunction were anticipated for humans. Nevertheless, few clinical studies have confirmed its real-world efficacy on hepatic dysfunction. This trial assessed the efficacy and safety of luseogliflozin in patients with T2DM complicated by hepatic dysfunction.

Methods: This prospective, single-site, singlearm, open-label, exploratory trial included 55 subjects with T2DM complicated by hepatic dysfunction. Subjects were administered luseogliflozin and observed for 52 weeks. The primary endpoints were the change in aspartate aminotransferase (AST), alanine aminotransferase (ALT), gamma-glutamyl transpeptidase $(\gamma$-GTP), and hemoglobin A1c (HbA1c) from baseline to week 52 . The secondary endpoints included body weight, body mass index (BMI), waist circumference, blood pressure, fasting plasma glucose (FPG), homeostatic model
\end{abstract}

H. Seino ( $\square)$

Seino Internal Medical Clinic, Kaisei, Koriyama,

Fukushima, Japan

e-mail: kn7jh5@bma.biglobe.ne.jp assessment beta (HOMA- $\beta$ ), homeostatic model assessment of insulin resistance (HOMA-IR), ferritin, Mac-2 binding protein (M2-BP), fatty liver index (FLI), fibrosis-4 (FIB-4) index, type IV collagen 7S domain, nonalcoholic fatty liver disease (NAFLD) fibrosis score, high-sensitivity C-reactive protein (hs-CRP), and interleukin-6 (IL-6).

Results: AST, ALT, $\gamma$-GTP, and HbA1c significantly decreased from baseline to week 52. Body weight, BMI, waist circumference, and FPG also significantly decreased. HOMA-IR significantly decreased but HOMA- $\beta$ was unchanged. FLI, ferritin, M2-BP, and NAFLD fibrosis scores significantly decreased whereas the FIB-4 index and type IV collagen 7S domain did not significantly change. The hs-CRP and IL-6 levels did not significantly change.

Conclusion: Luseogliflozin administration in patients with T2DM complicated by hepatic dysfunction was well tolerated, did not worsen the hepatic condition, and might even be beneficial to improve hepatic function, reduce liver fat, and attenuate liver injury and fibrosis.

Trial Registration: This study was registered under the University Hospital Medical Information Network Clinical Trial Registry (UMINCTR) (No. UMIN000025808) and the Japan Registry of Clinical Trials (jRCT) (No. jRCTs021180017). 
Keywords: Hepatic Luseogliflozin; SGLT2 inhibitor; Type 2 diabetes mellitus

\section{Key Summary Points}

Why carry out this study?

Favorable effects of the sodium-glucose co-transporter inhibitor luseogliflozin on hepatic dysfunction are expected in humans.

Few clinical studies have confirmed the real-world efficacy of luseogliflozin in patients with type 2 diabetes mellitus complicated by hepatic dysfunction.

This trial assessed the efficacy and safety of luseogliflozin in patients with type 2 diabetes mellitus complicated by hepatic dysfunction.

\section{What was learned from the study?}

Luseogliflozin administration in patients with T2DM and hepatic dysfunction was well tolerated, did not worsen the hepatic condition, and might even be beneficial to improve hepatic function, reduce liver fat, and attenuate liver injury and fibrosis.

\section{DIGITAL FEATURES}

This article is published with digital features, including a summary slide, to facilitate understanding of the article. To view digital features for this article go to https://doi.org/10.6084/ m9.figshare.13643351.

\section{INTRODUCTION}

Previous studies have associated type 2 diabetes mellitus (T2DM) with hepatic dysfunction. The association between T2DM and hepatic dysfunction is classified as follows [1, 2]: (1) hepatic dysfunction caused by T2DM (nonalcoholic fatty liver disease (NAFLD), nonalcoholic steatohepatitis (NASH), diabetic hepatopathy, or glycogenic hepatopathy exacerbated by T2DM); (2) T2DM caused by hepatic dysfunction (hepatic diabetes); and (3) hepatic dysfunction simultaneous with T2DM (chronic autoimmune hepatitis and autoimmune hepatic dysfunction). The prevalence of NAFLD in patients with T2DM was as high as $50-70 \%$ $[3,4]$. Hepatic dysfunction was a major cause of death in patients with T2DM [5-7]. Therefore, improvements in glycemic control and hepatic function are clinically important goals in the treatment of patients with T2DM complicated by hepatic dysfunction.

Novel oral hypoglycemic agents known as sodium-glucose co-transporter 2 (SGLT2) inhibitors have been recently launched. They are widely used in T2DM treatment. SGLT2 inhibitors block renal glucose reabsorption, promote urinary glucose excretion, and lower plasma glucose levels in an insulin-independent manner. Hence, there is a reduced risk of induction of hypoglycemia associated with them. The safety of SGLT2 inhibitors in combination with other hypoglycemic agents has been confirmed [8]. Moreover, SGLT2 inhibitors confer cardiovascular protection $[9,10]$, reduce body weight [11], lower mean 24-h glucose levels [12], decrease blood pressure [13], and improve blood lipid factors [14].

Luseogliflozin is a type of SGLT2 inhibitor. It improved NASH in an animal T2DM model [15]. The favorable effects of luseogliflozin on hepatic dysfunction could be expected also in humans. Nevertheless, few clinical studies have confirmed its real-world efficacy on hepatic dysfunction $[8,14,16]$. Therefore, the aim of this study was to evaluate luseogliflozin efficacy and safety in patients with T2DM complicated by hepatic dysfunction.

\section{METHODS}

\section{Study Design}

A single-center, single-arm, open-label, prospective interventional, exploratory trial was conducted from November 2016 to September 
2020. It was registered at the University Hospital Medical Information Network Clinical Trial Registry (UMIN-CTR/registration No. UMIN000025808) and the Japan Registry of Clinical Trials (jRCT/registration No. jRCTs021180017). The study protocol was approved by the Institutional Review Board of Seino Internal Medical Clinic in November 2016 according to the Ethical Guidelines for Medical and Health Research Involving Human Subjects issued by the Ministry of Health, Labour and Welfare in Japan. As the Clinical Trials Act was dispensed, the study protocol was reinspected and approved by the Fukushima Medical University Certified Review Board and certification was procured from the Minister of Health, Labour and Welfare in Japan in March 2019. The study was conducted in accordance with the Declaration of Helsinki, the Ethical Guidelines for Medical and Health Research Involving Human Subjects issued by the Ministry of Health, Labour and Welfare in Japan, the Clinical Trials Act, and other current legal regulations in Japan. Written informed consent was obtained from all participants after the study was fully explained to them. To minimize bias, data management and statistical analyses were conducted by third-party entities (DOT World Co. Ltd., Tokyo, Japan; Soiken Inc., Tokyo, Japan).

\section{Patient Population}

Patients with T2DM and hepatic dysfunction were included in the present trial. Patient inclusion criteria were (1) men and women aged at least 20 years and no greater than 80 years at the time of trial participation consent; (2) poor glycemic control despite diet and exercise therapy or treatment with hypoglycemic agents for at least 12 weeks; (3) HbA1c $\geq 6.5 \%$ and $\leq 9.5 \%$; (4) $\mathrm{BMI} \geq 20 \mathrm{~kg} / \mathrm{m}^{2}$; (5) provision of written informed consent; and (6) ALT $\geq 31 \mathrm{IU} /$ L. Patient exclusion criteria were (1) type 1 diabetes mellitus; (2) history of severe ketosis, diabetic coma, or precoma; (3) severe pre- or post-surgical infection or serious trauma; (4) estimated glomerular filtration rate (eGFR) $<30 \mathrm{~mL} / \mathrm{min} / 1.73 \mathrm{~m}^{2}$; (5) history of stroke, myocardial infarction, or other serious cardiovascular complications requiring hospitalization for no more than 6 months prior to giving trial participation consent; (6) existing use of SGLT2 inhibitor; (7) pregnancy, breastfeeding, possible pregnancy, or intention to become pregnant; (8) history of hypersensitivity to luseogliflozin or any of its components; and (9) any conditions deemed inappropriate by the physicians for trial participation.

\section{Study Intervention}

As this study was a single-arm trial, randomization was not conducted. The subjects furnished informed consent and then began to consume $2.5 \mathrm{mg}$ luseogliflozin orally once daily either before or after breakfast. The study intervention was conducted for 52 weeks and the subjects were observed at baseline (week 0 ) and at weeks 12, 24, and 52. All enrolled subjects were prohibited from using any SGLT2 inhibitor other than luseogliflozin during the trial. Moreover, enrolled subjects were not permitted to change the type, usage, or dose of any other therapeutic agents such as antiplatelet, antihypertensive, or antidyslipidemia drugs. They were not allowed to alter the type or degree of diet and exercise therapy during the study, provided that these treatments were safely regulated. Nevertheless, if the study subjects met the following criteria for discontinuation during the study period, the study intervention was discontinued; occurrence of adverse events that make the continuation of the study intervention difficult, occurrence of severe hypoglycemia, visit discontinuation or change of place of residence of the subject, consent withdrawal, use of prohibited concomitant agents, or other conditions that the attending physician considers to be inappropriate to continue the study intervention.

The medical examination by interview, blood collection, urine collection, and measurements of body weight, body mass index (BMI), waist circumference, blood pressure, and pulse were conducted at each observation point according to the observation schedule prespecified in the study protocol. Regarding the 
subjects' background, drinking habit and smoking habit of the study subjects were investigated by the medical examination by interview, asking drink or smoke frequency as follows: none, once/weekly, 2-3 times/weekly, and 4 times or more/weekly.

\section{Study Outcomes}

The primary endpoints were the change in aspartate aminotransferase (AST), alanine aminotransferase (ALT), gamma-glutamyl transpeptidase $(\gamma-\mathrm{GTP})$, and hemoglobin A1c (HbA1c) level from baseline to week 52. The secondary endpoints were (1) changes in AST, ALT, $\gamma$-GTP, and HbA1c levels from baseline to weeks 12 and 24; (2) changes in fasting plasma glucose (FPG) level, homeostatic model assessment beta (HOMA- $\beta$ ), homeostatic model assessment of insulin resistance (HOMA-IR), body weight, BMI, waist circumference, blood pressure, NAFLD fibrosis score, fibrosis-4 (FIB-4) index, and fatty liver index (FLI) from baseline to weeks 12,24 , and 52 ; (3) changes in type IV collagen 7S domain, ferritin, Mac- 2 binding protein (M2-BP), and high-sensitivity C-reactive protein (hs-CRP)) level from baseline to weeks 24 and 52; and (4) change in interleukin-6 (IL-6) level from baseline to week 52 .

\section{Sample Size Calculation and Statistical Analysis}

Since this study was planned as an exploratory study, the target number of enrolled subjects was defined to be 50 , based on the possible number of subjects who could give their consent following daily medical examinations at the Seino Internal Medical Clinic.

The primary and secondary endpoints were evaluated on the full analysis set (FAS) which includes all subjects assigned to a study intervention. However, subjects who did not receive the study agent were excluded from the FAS. Subjects for whom no data related to the efficacy endpoints could be obtained after study agent initiation were also excluded from the FAS. The per-protocol set (PPS) excluded subjects from the FAS if they presented with substantial protocol violations such as eligible criteria nonconformance, use of prohibited drugs, and poor adherence to the study agent. The safety analysis included all treated patients. All two-sided tests were performed and $P<0.05$ was considered as statistically significant. Summary statistics (number of subjects, mean, standard deviation, minima, median, and maxima) and changes from baseline were calculated for continuous data. A one-sample $t$ test was conducted to identify the change from baseline for intragroup before-and-after comparison. Frequencies and proportions were calculated for the categorical data. Multiple logistic regression analysis was performed to test the relationship between the change in HbA1c and other factors from baseline to week 52. First, by using univariate regression analysis, variables which had a $P$ value less than 0.05 were detected. By using these factors as explanatory variables, multiple regression analysis was performed. SAS version 9.4 (SAS, Cary, NC, USA) was used to perform all statistical analyses.

\section{RESULTS}

\section{Baseline Characteristics of Study Participants}

Between April 2017 and September 2018, 55 subjects were enrolled in this study and received the intervention (luseogliflozin administration). One subject discontinued the visit to the institution during the study. Five subjects discontinued luseogliflozin use because of adverse events during the study. Hence, 55 subjects were included in both the safety analysis set and the FAS (Fig. 1) and 49 subjects completed the study intervention. The baseline characteristics of the subjects are summarized in Table 1.

\section{Primary Endpoints}

The primary endpoints of this study were the change in AST, ALT, $\gamma$-GTP, and HbA1c levels from baseline to week 52. The AST, ALT, $\gamma$-GTP, and HbA1c significantly decreased from 


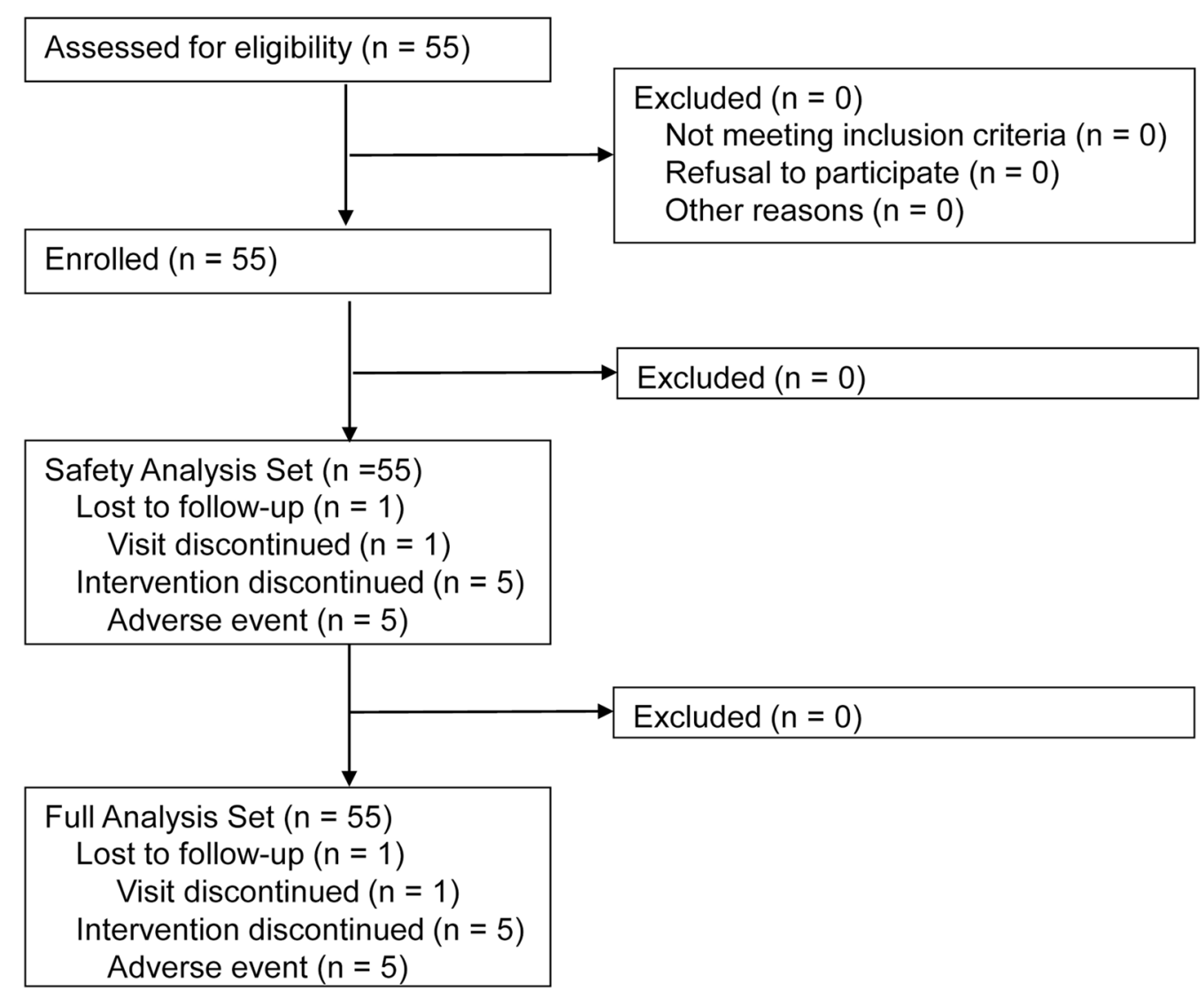

Fig. 1 Flowchart depicting study participant enrollment, allocation, and analysis. All 55 eligible subjects enrolled in this study were included and analyzed in the safety analysis set and full analysis set

baseline to week 52 (Fig. 2). The changes in AST, ALT, $\gamma$-GTP, and HbA1c from baseline to week 52 were $-12.2 \pm 13.0 \mathrm{IU} / \mathrm{L}(P<0.0001)$, $-25.2 \pm 23.9 \mathrm{IU} / \mathrm{L}(P<0.0001),-21.4 \pm 22.1$ IU/L $\quad(P<0.0001), \quad$ and $\quad-0.6 \% \pm 0.5 \%$ $(P<0.0001)$, respectively. The AST, ALT, $\gamma$-GTP, and HbA1c levels also significantly decreased from baseline to weeks 12 and 24 (Fig. 2). The changes in AST level from baseline were $-8.9 \pm 11.1 \mathrm{IU} / \mathrm{L} \quad(P<0.0001)$ and $-10.5 \pm$ $13.9 \mathrm{IU} / \mathrm{L} \quad(P<0.0001)$ at weeks 12 and 24 , respectively. The changes in ALT level from baseline were $-17.5 \pm 19.4 \mathrm{IU} / \mathrm{L}(P<0.0001)$ and $-21.0 \pm 26.0 \mathrm{IU} / \mathrm{L}(P<0.0001)$ at weeks 12 and 24 , respectively. The changes in $\gamma$-GTP level from baseline were $-12.5 \pm 19.1 \mathrm{IU} / \mathrm{L}$ $(P<0.0001) \quad$ and $\quad-12.8 \pm 26.7 \mathrm{IU} / \mathrm{L} \quad(P=$ $0.0012)$ at weeks 12 and 24, respectively. The changes in HbA1c level from baseline were $-0.5 \% \pm 0.4 \% \quad(P<0.0001) \quad$ and $-0.5 \% \pm$
$0.5 \% \quad(P<0.0001)$ at weeks 12 and 24 , respectively.

\section{Hepatic Function Biomarkers}

FLI is a surrogate liver fat marker. FLI significantly decreased from baseline to weeks 12, 24 and 52 (Fig. 3).

$\mathrm{M} 2-\mathrm{BP}$ is a surrogate hepatic fibrosis marker. M2-BP level decreased from baseline to weeks 24 and 52 (Fig. 3). The changes in M2BP level from baseline were $-0.06 \pm 0.18$ $(P=0.0187)$ and $-0.08 \pm 0.18 \quad(P=0.0027)$ at weeks 24 and 52, respectively.

NAFLD fibrosis score, FIB-4 index, and type IV collagen $7 \mathrm{~S}$ domain are also hepatic fibrosis markers. The NAFLD fibrosis score significantly decreased from baseline to weeks 12 and 24 (Table 2). However, there was no significant change in NAFLD fibrosis score by 
Table 1 Baseline characteristics of subjects

\begin{tabular}{ll}
\hline Characteristics & $\begin{array}{l}\text { Mean } \pm \text { standard deviation } \\
\text { or } \boldsymbol{n}(\%)\end{array}$ \\
\hline Age (years) & $52.7 \pm 11.4$ \\
Gender (male/female) & $38(69.1) / 17(30.9)$ \\
Height (cm) & $165.6 \pm 8.3$ \\
Body weight (kg) & $80.4 \pm 14.9$ \\
BMI (kg/m ${ }^{2}$ ) & $29.2 \pm 4.0$ \\
Waist circumstance & $98.5 \pm 9.9$ \\
(cm) & \\
Duration of diabetes & $8.8 \pm 6.4$ \\
mellitus & \\
Drinking habit & $36(65.5)$ \\
Smoking habit & $24(43.6)$ \\
Cerebrovascular & $3(5.5)$ \\
complications & \\
Diabetic complications & $20(36.4)$ \\
Diabetic nephropathy & $14(25.5)$ \\
Diabetic neuropathy & $4(7.3)$ \\
Diabetic retinopathy & $5(9.1)$ \\
Hypertension & $30(54.5)$ \\
Dyslipidemia & $29(52.7)$ \\
Antidiabetic agents & $13(26)$ \\
Sulfonylurea & $11(20.0)$ \\
Biguanide & $49(89.1)$ \\
A-Glucosidase & $6(10.9)$ \\
inhibitor & \\
Glinide & $1(1.8)$ \\
Thiazolidinedione & $1(1.8)$ \\
DPP4 inhibitor & $30(54.5)$ \\
SGLT2 inhibitor & $0(0)$ \\
\hline
\end{tabular}

Table 1 continued

\begin{tabular}{ll}
\hline Characteristics & $\begin{array}{l}\text { Mean } \mathbf{\pm} \text { standard deviation } \\
\text { or } \boldsymbol{n}(\%)\end{array}$ \\
\hline $\begin{array}{l}\text { Lipid-lowering agent } \\
\text { Antihyperuricemic agent }\end{array}$ & $4(40.0)$ \\
$\begin{array}{l}\text { Other concomitant } \\
\text { agents }\end{array}$ & $36(65.5)$ \\
\hline
\end{tabular}

Data are presented as mean \pm standard deviation or $n(\%)$ among 55 subjects in the full analysis set

$B M I$ body mass index, DPP4 dipeptidyl peptidase-4, SGLT2 sodium-glucose co-transporter protein 2, GLP-1 glucagon-like peptide 1

week 52. The FIB-4 index and the type IV collagen $7 \mathrm{~S}$ domain did not significantly change from baseline.

Ferritin is a hepatic impairment biomarker. Its levels significantly decreased from baseline to weeks 24 and 52 (Table 2).

\section{Inflammation Biomarkers}

The hs-CRP and IL-6 levels did not significantly change from baseline either to week 24 and 52 (Table 2).

\section{Vital Signs}

Body weight, BMI, and waist circumstance significantly decreased from baseline to weeks 12, 24 , and 52 (Table 2). In contrast, the systolic blood pressure significantly decreased only from baseline to week 12 and significant decreases disappeared after week 24 . No significant changes were observed for diastolic blood pressure or pulse.

\section{Glucose Metabolism}

The fasting plasma glucose levels significantly decreased from baseline to weeks 12, 24, and 52 (Table 2). Plasma insulin and HOMA-IR 

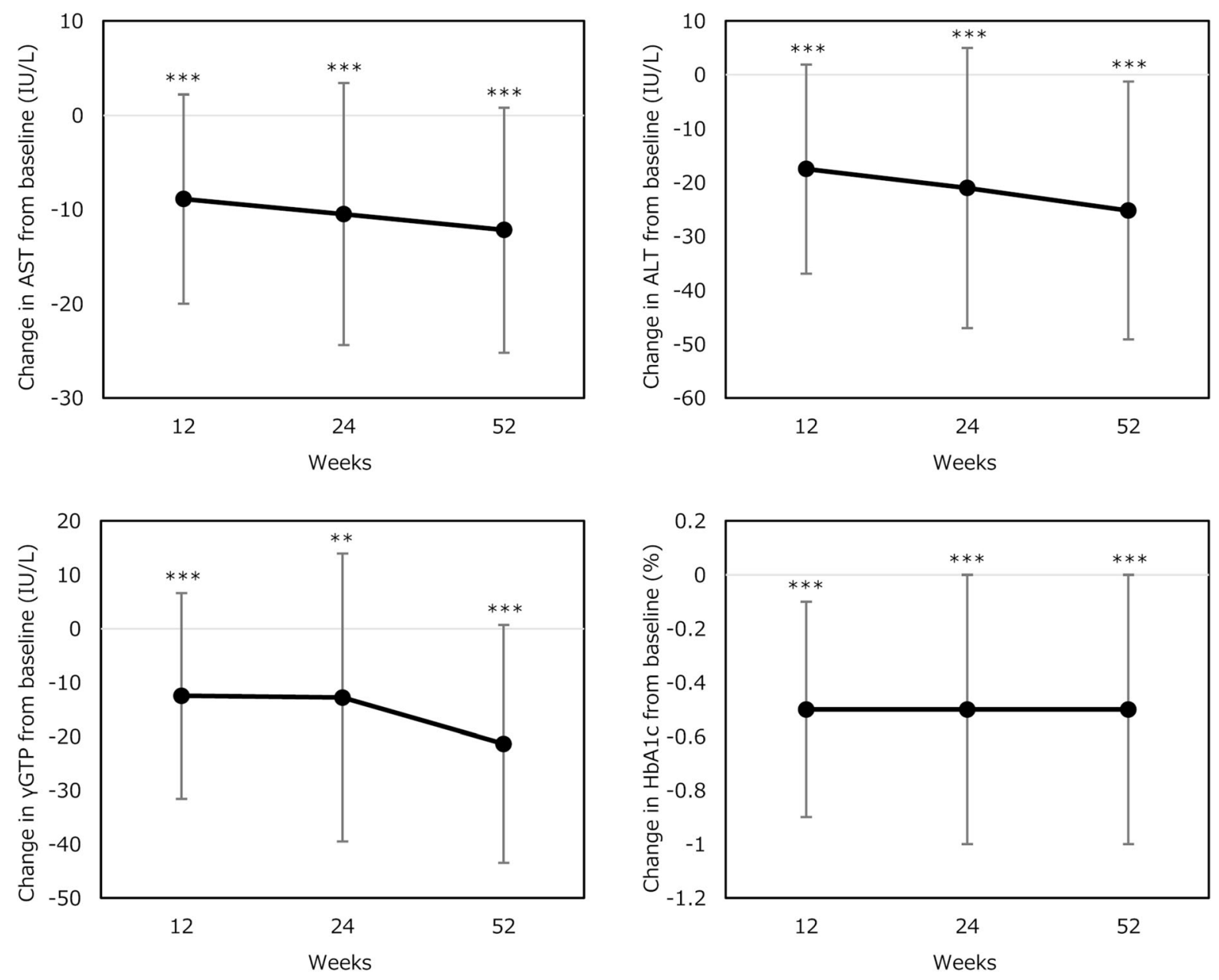

Fig. 2 Change from baseline in the primary endpoints (AST, ALT, $\gamma$-GTP, and HbA1c). Data are represented as mean \pm standard deviation. ${ }^{*} P<0.05$; ${ }^{* *} P<0.01$; and ${ }^{* * *} P<0.001, \quad$ respectively. AST aspartate

aminotransferase, ALT alanine aminotransferase, $\gamma$-GTP gamma-glutamyl transpeptidase, HbAlc hemoglobin Alc

significantly decreased from baseline to weeks 12,24 , and 52. HOMA- $\beta$ did not significantly change from baseline.

\section{Correlation Between Change in HbA1c and Other Factors}

By univariate regression analysis, the changes in AST, M2-BP, white blood cell (WBC), and type IV collagen $7 \mathrm{~S}$ domain from baseline to week 52 were significantly correlated with the change in HbA1c from baseline to week 52 . Using these factors as explanatory variables, multiple regression analysis was performed.

Change in AST and type IV collagen 7S domain from baseline to week 52 were significantly correlated with the change in HbA1c from baseline to week 52 (Table 3).

\section{Safety Outcomes}

Twenty-four adverse events were reported in 18 out of 55 subjects $(32.7 \%)$ (Table 4$)$. The most common adverse events were genital infection and dry skin (six subjects each; 10.9\%). Four serious adverse events (facial paresis, venous thrombosis, Baker's cyst, and gastric cancer) were reported during the study. Nevertheless, 

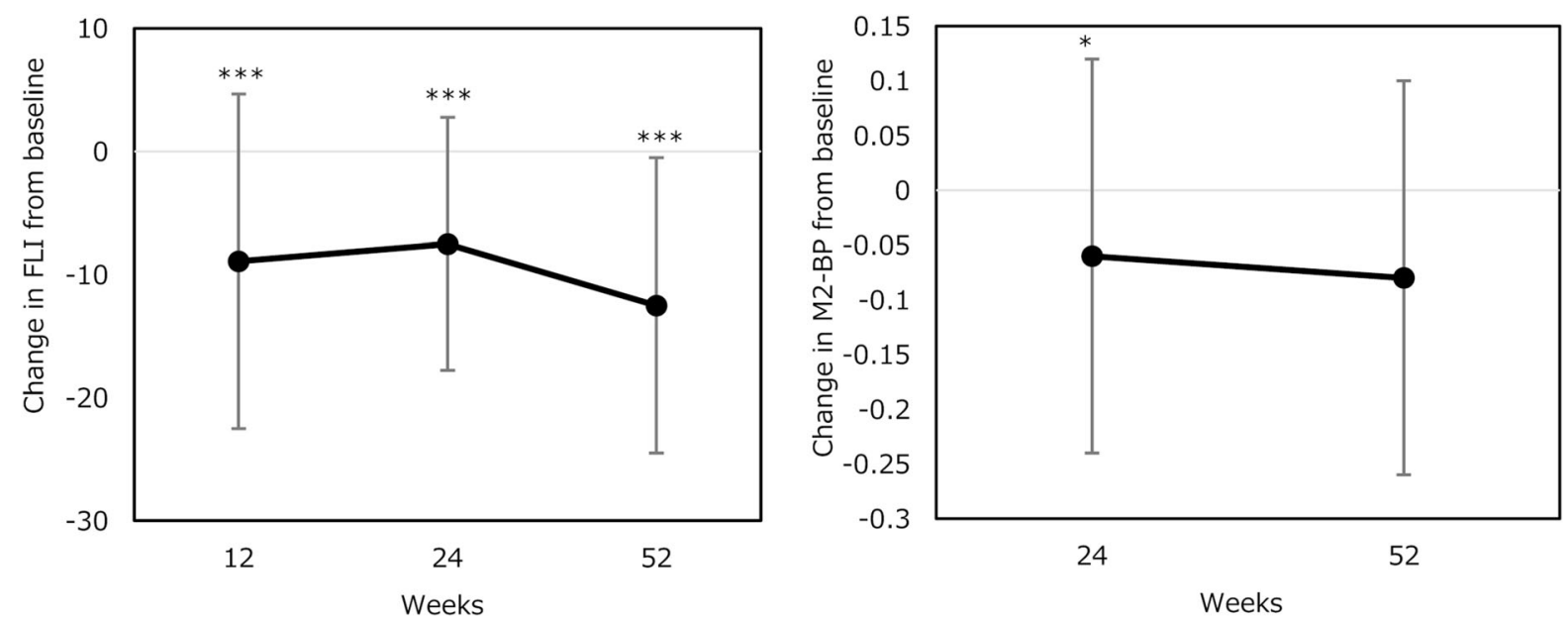

Fig. 3 Change from baseline in hepatic function biomarkers (FLI and M2-BP). Data are represented as mean \pm standard deviation. ${ }^{*} P<0.05$; ${ }^{* *} P<0.01$; and ${ }^{* *} P<0.001$, respectively. FLI fatty liver index, M2-BP Mac-2 binding protein

none of them was considered to be related to luseogliflozin administration. No hypoglycemia or severe hypoglycemia was reported during the study.

\section{DISCUSSION}

The purpose of this study was to assess the efficacy and safety of the SGLT2 inhibitor luseogliflozin in patients with T2DM complicated by hepatic dysfunction. We observed improvement in glycemic control and the hepatic function biomarkers AST, ALT, and $\gamma$-GTP. Moreover, FLI (a surrogate liver fat marker), ferritin (a hepatic impairment biomarker), M2$\mathrm{BP}$, and the NAFLD fibrosis score (hepatic fibrosis biomarkers) were significantly improved in response to luseogliflozin administration.

Several previous studies in patients with T2DM showed that luseogliflozin administration decreased hepatic function biomarkers $[8,14,16]$. A recent single-arm LEAD trial in patients with T2DM and NAFLD demonstrated a significant decrease in hepatic function biomarkers [17]. The results of the present study were consistent with those of previous reports. The present study provided supportive evidence that luseogliflozin improved hepatic function in patients with T2DM complicated by hepatic dysfunction.
Additionally, this study revealed improvement in the hepatic fibrosis biomarkers M2-BP, NAFLD fibrosis score, and ferritin following luseogliflozin administration in patients with T2DM complicated by hepatic dysfunction. However, FIB-4 index score and type IV collagen 7S did not change significantly after luseogliflozin administration. The LEAD trial showed decrease in the AST, ALT, $\gamma$-GTP, and ferritin levels in patients with T2DM with NAFLD; however, the hepatic fibrosis markers FIB-4 index, NAFLD fibrosis score, type IV collagen 7S, and M2-BP were unchanged [17]. Hence, luseogliflozin may only be able to ameliorate hepatic dysfunction but not established NAFLD.

The beneficial effects of SGLT2 inhibitors on body weight and composition have been reported [18-21]. The present study demonstrated significant reduction in body weight, BMI, and waist circumstance after luseogliflozin administration. The surrogate liver fat marker FLI significantly decreased in the present study. This finding was consistent with that reported by previous studies which demonstrated decrease in liver fat following SGLT2 inhibitor treatment [22-24] and might explain the hepatic function improvement observed here.

This trial also disclosed that plasma insulin levels and HOMA-IR were significantly decreased by luseogliflozin administration. This finding was consistent with earlier reports of 
Table 2 Clinical outcomes

\begin{tabular}{|c|c|c|c|c|c|c|}
\hline \multirow[t]{2}{*}{ Endpoint } & \multirow[t]{2}{*}{ Week } & \multicolumn{2}{|c|}{ Measurement } & \multicolumn{3}{|c|}{ Change } \\
\hline & & $n$ & Mean $\pm S D$ & $n$ & Mean $\pm S D$ & $P$ value \\
\hline \multirow[t]{4}{*}{ NAFLD fibrosis score } & 0 & 55 & $4.1 \pm 0.6$ & & & \\
\hline & 12 & 54 & $4.0 \pm 0.8$ & 54 & $-0.2 \pm 0.5$ & 0.0225 \\
\hline & 24 & 52 & $3.9 \pm 0.6$ & 52 & $-0.5 \pm 0.4$ & 0.0005 \\
\hline & 52 & 50 & $4.0 \pm 0.7$ & 50 & $-0.1 \pm 0.6$ & 0.1178 \\
\hline \multirow[t]{4}{*}{ FIB-4 index } & 0 & 55 & $3.1 \pm 1.8$ & & & \\
\hline & 12 & 54 & $3.1 \pm 1.5$ & 54 & $-0.1 \pm 0.9$ & 0.6897 \\
\hline & 24 & 51 & $3.0 \pm 1.4$ & 51 & $-0.1 \pm 0.8$ & 0.3621 \\
\hline & 52 & 47 & $3.2 \pm 1.6$ & 47 & $0.2 \pm 0.9$ & 0.0774 \\
\hline \multirow[t]{3}{*}{ Type IV collagen $7 \mathrm{~S}$ domain $(\mathrm{ng} / \mathrm{mL})$} & 0 & 55 & $4.6 \pm 1.2$ & & & \\
\hline & 24 & 52 & $4.6 \pm 0.8$ & 52 & $0.0 \pm 1.0$ & 0.8387 \\
\hline & 52 & 49 & $4.6 \pm 0.7$ & 49 & $0.1 \pm 1.0$ & 0.4492 \\
\hline \multirow[t]{3}{*}{ Ferritin $(\mathrm{ng} / \mathrm{mL})$} & 0 & 55 & $191.4 \pm 144.9$ & & & \\
\hline & 24 & 52 & $123.9 \pm 113.2$ & 52 & $-64.6 \pm 73.1$ & $<0.0001$ \\
\hline & 52 & 49 & $113.6 \pm 97.9$ & 49 & $-76.9 \pm 74.2$ & $<0.0001$ \\
\hline \multirow[t]{3}{*}{ hs-CRP (mg/dL) } & 0 & 55 & $0.11 \pm 0.13$ & & & \\
\hline & 24 & 52 & $0.09 \pm 0.10$ & 52 & $-0.02 \pm 0.10$ & 0.1222 \\
\hline & 52 & 49 & $0.08 \pm 0.11$ & 49 & $-0.03 \pm 0.13$ & 0.0968 \\
\hline \multirow[t]{2}{*}{ IL-6 (pg/mL) } & 0 & 55 & $2.1 \pm 2.7$ & & & \\
\hline & 52 & 49 & $1.6 \pm 1.2$ & 49 & $-0.3 \pm 1.1$ & 0.0847 \\
\hline \multirow[t]{4}{*}{ Body weight (kg) } & 0 & 55 & $80.4 \pm 14.9$ & & & \\
\hline & 12 & 53 & $77.5 \pm 14.0$ & 53 & $-1.9 \pm 1.3$ & $<0.0001$ \\
\hline & 24 & 52 & $78.0 \pm 14.0$ & 52 & $-2.6 \pm 1.8$ & $<0.0001$ \\
\hline & 52 & 50 & $77.6 \pm 14.2$ & 50 & $-3.1 \pm 2.5$ & $<0.0001$ \\
\hline \multirow[t]{4}{*}{ BMI $\left(\mathrm{kg} / \mathrm{m}^{2}\right)$} & 0 & 55 & $29.2 \pm 4.0$ & & & \\
\hline & 12 & 53 & $28.3 \pm 4.0$ & 53 & $-0.7 \pm 0.5$ & $<0.0001$ \\
\hline & 24 & 52 & $28.3 \pm 3.8$ & 52 & $-0.9 \pm 0.7$ & $<0.0001$ \\
\hline & 52 & 50 & $28.0 \pm 3.8$ & 50 & $-1.1 \pm 0.9$ & $<0.0001$ \\
\hline \multirow[t]{4}{*}{ Waist circumstance $(\mathrm{cm})$} & 0 & 55 & $98.5 \pm 9.9$ & & & \\
\hline & 12 & 53 & $97.0 \pm 10.2$ & 53 & $-1.6 \pm 3.0$ & 0.0003 \\
\hline & 24 & 52 & $97.2 \pm 9.9$ & 52 & $-1.6 \pm 3.2$ & 0.0006 \\
\hline & 52 & 47 & $96.7 \pm 10.3$ & 47 & $-2.4 \pm 3.2$ & $<0.0001$ \\
\hline Systolic blood pressure (mm Hg) & 0 & 55 & $131.9 \pm 11.9$ & & & \\
\hline
\end{tabular}


Table 2 continued

\begin{tabular}{|c|c|c|c|c|c|c|}
\hline \multirow[t]{2}{*}{ Endpoint } & \multirow[t]{2}{*}{ Week } & \multicolumn{2}{|c|}{ Measurement } & \multicolumn{3}{|c|}{ Change } \\
\hline & & $n$ & Mean \pm SD & $n$ & Mean \pm SD & $P$ value \\
\hline & 12 & 53 & $127.4 \pm 11.6$ & 53 & $-4.4 \pm 13.0$ & 0.0172 \\
\hline & 24 & 52 & $130.5 \pm 11.2$ & 52 & $-1.0 \pm 13.2$ & 0.6019 \\
\hline & 52 & 50 & $129.8 \pm 12.5$ & 50 & $-1.9 \pm 14.6$ & 0.3565 \\
\hline \multirow[t]{4}{*}{ Diastolic blood pressure $(\mathrm{mm} \mathrm{Hg})$} & 0 & 55 & $81.3 \pm 9.5$ & & & \\
\hline & 12 & 53 & $79.2 \pm 8.8$ & 53 & $-2.1 \pm 9.4$ & 0.1178 \\
\hline & 24 & 52 & $78.7 \pm 8.7$ & 52 & $-2.4 \pm 9.4$ & 0.0731 \\
\hline & 52 & 50 & $79.8 \pm 8.5$ & 50 & $-1.4 \pm 10.2$ & 0.3223 \\
\hline \multirow[t]{4}{*}{ Pulse } & 0 & 53 & $75.0 \pm 10.7$ & & & \\
\hline & 12 & 52 & $74.3 \pm 11.8$ & 50 & $-0.3 \pm 9.7$ & 0.8163 \\
\hline & 24 & 52 & $73.7 \pm 11.6$ & 50 & $-1.3 \pm 8.6$ & 0.2809 \\
\hline & 52 & 48 & $73.8 \pm 12.0$ & 46 & $-0.8 \pm 8.1$ & 0.4930 \\
\hline \multirow[t]{4}{*}{ Fasting plasma glucose $(\mathrm{mg} / \mathrm{dL})$} & 0 & 55 & $148.8 \pm 20.7$ & & & \\
\hline & 12 & 54 & $131.3 \pm 17.7$ & 54 & $-17.9 \pm 15.5$ & $<0.0001$ \\
\hline & 24 & 52 & $133.2 \pm 23.5$ & 52 & $-16.8 \pm 20.2$ & $<0.0001$ \\
\hline & 52 & 50 & $130.4 \pm 19.8$ & 50 & $-18.8 \pm 19.5$ & $<0.0001$ \\
\hline \multirow[t]{4}{*}{ Plasma insulin $(\mu \mathrm{U} / \mathrm{mL})$} & 0 & 55 & $12.8 \pm 7.0$ & & & \\
\hline & 12 & 53 & $10.9 \pm 5.7$ & 53 & $-2.0 \pm 4.6$ & 0.0026 \\
\hline & 24 & 52 & $11.0 \pm 5.8$ & 52 & $-2.0 \pm 5.0$ & 0.0065 \\
\hline & 52 & 49 & $11.1 \pm 6.9$ & 49 & $-1.7 \pm 5.4$ & 0.0323 \\
\hline \multirow[t]{4}{*}{ НОМА- $\beta(\%)$} & 0 & 55 & $55.6 \pm 30.1$ & & & \\
\hline & 12 & 53 & $59.3 \pm 28.3$ & 53 & $3.8 \pm 25.3$ & 0.2802 \\
\hline & 24 & 52 & $61.0 \pm 32.1$ & 52 & $5.7 \pm 29.4$ & 0.1710 \\
\hline & 52 & 49 & $61.5 \pm 35.3$ & 49 & $6.8 \pm 26.0$ & 0.0714 \\
\hline \multirow[t]{4}{*}{ HOMA-IR } & 0 & 55 & $4.8 \pm 2.7$ & & & \\
\hline & 12 & 53 & $3.6 \pm 2.1$ & 53 & $-1.2 \pm 1.7$ & $<0.0001$ \\
\hline & 24 & 52 & $3.7 \pm 2.2$ & 52 & $-1.2 \pm 1.8$ & $<0.0001$ \\
\hline & 52 & 49 & $3.6 \pm 2.4$ & 49 & $-1.1 \pm 2.2$ & 0.0007 \\
\hline
\end{tabular}

Data are presented as $n$ and mean \pm standard deviation

NAFLD fibrosis score nonalcoholic fatty liver disease fibrosis score, FIB-4 fibrosis-4, hs-CRP high-sensitivity C-reactive protein, $I L-6$ interleukin-6, BMI body mass index, HOMA- $\beta$ homeostatic model assessment beta, HOMA-IR homeostatic model assessment of insulin resistance 
Table 3 Correlation between change in HbAlc and other factors from baseline to week 52

\begin{tabular}{lcc}
\hline Factor & Partial regression coefficient $\mathbf{9 5 \%}$ confidence interval $)$ & $\boldsymbol{P}$ value \\
\hline AST & $0.0110(0.0014$ to 0.0205$)$ & 0.0254 \\
M2-BP & $0.5722(-0.1118$ to 1.2563$)$ & 0.0987 \\
WBC & $-0.0098(-0.0200$ to 0.0004$)$ & 0.0599 \\
Type IV collagen 7S domain & $0.1304(0.0115$ to 0.2493$)$ & 0.0324 \\
\hline
\end{tabular}

Data are presented as partial regression coefficient ( $95 \%$ confidence interval)

$A S T$ aspartate aminotransferase, $M 2-B P$ Mac-2 binding protein, $W B C$ white blood cell

Table 4 Adverse events

\begin{tabular}{lc}
\hline Adverse event & Frequency \\
\hline Death & $0(0)$ \\
Any adverse events & $18(32.7)$ \\
Serious adverse event & $4(7.3)$ \\
Genital infection & $6(10.9)$ \\
Dry skin & $6(10.9)$ \\
Balanitis & $1(1.8)$ \\
Dehydration & $1(1.8)$ \\
Skin rash & $1(1.8)$ \\
Constipation & $1(1.8)$ \\
Genital itching & $1(1.8)$ \\
Facial paresis & $1(1.8)$ \\
Arthritis & $1(1.8)$ \\
Disc herniation (low back pain) & $1(1.8)$ \\
Venous thrombosis & $1(1.8)$ \\
Pulled muscle & $1(1.8)$ \\
Baker's cyst & $1(1.8)$ \\
Gastric cancer & $1(1.8)$ \\
\hline Data &
\end{tabular}

Data are presented as $n(\%)$

reductions in HOMA-IR (insulin resistance improvement) by SGLT2 inhibitors $[25,26]$. In contrast, HOMA- $\beta$ did not significantly improve in the present study. SGLT2 inhibitors apparently improved pancreatic $\beta$-cell function in animal models [27]. However, no study has evaluated the effects of SGLT2 inhibitors on pancreatic $\beta$-cell function in humans. A Korean clinical study showed that a group responding well to SGLT2 inhibitors presented with relatively higher HOMA than the group responding poorly to SGLT2 inhibitors. Nevertheless, the HOMA- $\beta$ level was not associated with SGLT2 inhibitor responsiveness [28]. The results of this study suggested that SGLT2 inhibitors improved insulin resistance but not insulin secretion in the pancreatic $\beta$-cells of patients with T2DM.

Here, luseogliflozin administration did not improve inflammation because it did not lower the hs-CRP or IL-6 levels. It has been previously reported that SGLT2 inhibitors suppress inflammation in animal models $[29,30]$. However, only a few human trials reported an association between SGLT2 inhibitors and inflammation [31, 32]. Further studies are needed to assess the efficacy of SGLT2 inhibitors in attenuation of inflammation.

By univariate regression analysis, the changes in AST, M2-BP, WBC, and type IV collagen $7 \mathrm{~S}$ domain from baseline to week 52 were significantly correlated with the change in HbA1c from baseline to week 52. By multiple regression analysis using these factors as explanatory variables, changes in AST and type IV collagen $7 \mathrm{~S}$ domain were again significant and independent determinants of change in HbA1c. It was reported that change in HbA1c was significantly associated with improvement of hepatic fibrosis [33]. Another study demonstrated that the fibrosis score were significantly improved in ALT responders [34]. Since the hepatic function biomarkers including AST, ALT, and $\gamma$-GTP, the glycemic control, and hepatic fibrosis 
biomarkers were improved by administration of luseogliflozin to patients with T2DM and hepatic dysfunction in this study, the results in this study were consistent with the previous reports. Inhibition of SGLT2 protein by administration of SGLT2 inhibitor causes improvement of insulin resistance and calorie loss by urinary glucose excretion, resulting enhancement of beta-oxidation, release of very low density lipoprotein (VLDL) from liver, and decrease in hepatic fat, hepatic lipotoxicity, hepatic inflammation, and hepatic fibrosis [35]. These might be the mechanism by which hepatic fibrosis was attenuated by administration of luseogliflozin in patients with T2DM and hepatic dysfunction.

No serious adverse events associated with luseogliflozin were detected in the present study. Genital infection and dry skin were the most commonly observed adverse reactions here but they are well-known side effects of SGLT2 inhibitors. There were no remarkable occurrences of any unknown side effects. No hypoglycemia was recorded during this trial. It was reported earlier that SGLT2 inhibitors lowered plasma glucose in an insulin-independent manner and posed a low risk of inducing hypoglycemia [36]. Moreover, recent large-scale clinical trials demonstrated that SGLT2 inhibitors prevented mortality, macrovascular complications, and renal impairment progression associated with T2DM $[9,10]$. Overall, SGLT2 inhibitors are well tolerated in patients with T2DM and hepatic dysfunction.

The study protocol described the enrollment of 50 subjects. Nevertheless, it was feasible for the Seino Internal Medical Clinic to support the enrollment of 55 subjects. The over-registration was reported to the Fukushima Medical University Certified Review Board and approved by them. The last five subjects were excluded and the results of the present study were reanalyzed with only 50 participants. Once again, all primary endpoints (ALT, AST, $\gamma$-GTP, and HbA1c) showed significant improvements. Therefore, over-registration by five patients did not influence the outcome of this trial and all 55 subjects were included in the FAS and analyzed.
This study had several limitations. First, there were relatively few enrolled subjects in this trial and it was conducted in only one clinical institution (Seino Internal Medical Clinic) in Japan. Second, it was a single-arm trial. Further larger confirmatory trials are required to confirm the effect of luseogliflozin in patients with T2DM complicated by hepatic dysfunction. Third, the eligibility criterion hepatic dysfunction was defined only by the ALT level $(\geq 31 \mathrm{IU} / \mathrm{L})$ at enrollment in this study to simplify the study design and to enroll the study subjects with similar disease condition. Further trials are required to confirm the effect of luseogliflozin in patients with T2DM complicated by hepatic dysfunction of other disease conditions. These constraints may limit the generalizability of this study and large-scale, multicenter randomized controlled trials are required to validate its findings.

\section{CONCLUSIONS}

Luseogliflozin administration in patients with T2DM complicated by hepatic dysfunction was tolerated, did not worsen the hepatic condition, and might even be beneficial to improve hepatic function, reduce liver fat, and attenuate liver injury and fibrosis. Further larger confirmatory trials are required to confirm the effect of luseogliflozin in patients with T2DM complicated by hepatic dysfunction.

\section{ACKNOWLEDGEMENTS}

The author thank the participants of the study and all clinical staff for their assistance in the execution of this trial.

Funding. This study was financially supported by Taisho Pharmaceutical Co. Ltd. The journal's Rapid Service Fee, and fee for the technical assistance in the launch and execution of the study and the medical writing of the manuscript by Soiken Inc. were also financially supported by Taisho Pharmaceutical Co. Ltd. 
Medical writing, Editorial, and Other Assistance. The author thanks DOT Worlds Co. Ltd. and Soiken Inc. for their technical assistance in the launch and execution of the study and the statistical analysis, and Arata Yoneda in Soiken Inc. for their support on the medical writing of the manuscript (funded by Taisho Pharmaceutical Co. Ltd).

Authorship. HS conceived and designed the study. HS enrolled the subjects, acquired the data, and drafted and revised the manuscript. HS met the International Committee of Medical Journal Editors (ICMJE) criteria for authorship of this article, assumed full responsibility for its overall integrity, and has given approval for this version to be published.

Disclosures. Hiroaki Seino received lecture fees from Shionogi Co. Ltd., Mitsubishi Tanabe Pharma Co., Astellas Pharma Inc., Novartis Pharma K.K., Eli Lilly and Co., Ono Pharmaceutical Co. Ltd., MSD K.K., and Sanofi K.K. Hiroaki Seino was awarded research grants from Taisho Pharmaceutical Co. Ltd., Takeda Pharmaceutical Co. Ltd., Novo Nordisk Pharma Ltd., Sanofi K.K., MSD K.K., Boehringer Ingelheim Pharmaceuticals, Inc., AstraZeneca K.K., Sanwa Kagaku Kenkyusho Co. Ltd., YL Biologics Ltd., and Japan Tobacco Inc.

Compliance with Ethics Guidelines. The study protocol was initially approved by the Institutional Review Board of the Seino Internal Medical Clinic in November 2016 according to the Ethical Guidelines for Medical and Health Research Involving Human Subjects issued by the Ministry of Health, Labour and Welfare in Japan. As the Clinical Trials Act was dispensed, the study protocol was reinspected and approved by the Fukushima Medical University Certified Review Board which obtained certification from the Minister of Health, Labour and Welfare in Japan in March 2019. The study was conducted in accordance with the Declaration of Helsinki, the Ethical Guidelines for Medical and Health Research Involving Human Subjects issued by the Ministry of Health, Labour and Welfare in Japan, the Clinical Trials Act, and other current legal regulations in Japan. Written informed consent was obtained from all participants after full explanation of the study.

Data Availability. The datasets generated during and/or analyzed during the current study are not publicly available due to the lack of a statement in the study protocol enabling data sharing with a third party after the end of the study and in the informed consent documents as well as lack of approval for data sharing by the Institutional Review Board of the Seino Internal Medical Clinic or the Fukushima Medical University Certified Review Board.

Open Access. This article is licensed under a Creative Commons Attribution-NonCommercial 4.0 International License, which permits any non-commercial use, sharing, adaptation, distribution and reproduction in any medium or format, as long as you give appropriate credit to the original author(s) and the source, provide a link to the Creative Commons licence, and indicate if changes were made. The images or other third party material in this article are included in the article's Creative Commons licence, unless indicated otherwise in a credit line to the material. If material is not included in the article's Creative Commons licence and your intended use is not permitted by statutory regulation or exceeds the permitted use, you will need to obtain permission directly from the copyright holder. To view a copy of this licence, visit http://creativecommons.org/licenses/by$\mathrm{nc} / 4.0 /$.

\section{REFERENCES}

1. Hamed AE, Elsahar M, Elwan NM, et al. Managing diabetes and liver disease association. Arab J Gastroenterol. 2018;19:166-79.

2. Hamed AE, Elwan N, Naguib M, et al. Diabetes association with liver diseases: an overview for clinicians. Endocr Metab Immune Disord Drug Targets. 2019;19:274-80.

3. Leite NC, Salles GF, Araujo AL, Villela-Nogueira CA, Cardoso CR. Prevalence and associated factors of non-alcoholic fatty liver disease in patients with type-2 diabetes mellitus. Liver Int. 2009;29:113-9. 
4. Chan WK, Tan AT, Vethakkan SR, Tah PC, Vijayananthan A, Goh KL. Non-alcoholic fatty liver disease in diabetics-prevalence and predictive factors in a multiracial hospital clinic population in Malaysia. J Gastroenterol Hepatol. 2013;28: 1375-83.

5. Tolman KG, Fonseca V, Dalpiaz A, Tan MH. Spectrum of liver disease in type 2 diabetes and management of patients with diabetes and liver disease. Diabetes Care. 2007;30:734-43.

6. Balkau B, Eschwege E, Ducimetiere P, Richard JL, Warnet JM. The high risk of death by alcohol related diseases in subjects diagnosed as diabetic and impaired glucose tolerant: the Paris Prospective Study after 15 years of follow-up. J Clin Epidemiol. 1991;44:465-74.

7. de Marco R, Locatelli F, Zoppini G, Verlato G, Bonora E, Muggeo M. Cause-specific mortality in type 2 diabetes. The Verona Diabetes Study. Diabetes Care. 1999;22:756-61.

8. Seino $\mathrm{Y}$, Inagaki $\mathrm{N}$, Haneda $\mathrm{M}$, et al. Efficacy and safety of luseogliflozin added to various oral antidiabetic drugs in Japanese patients with type 2 diabetes mellitus. J Diabetes Investig. 2015;6: 443-53.

9. Zinman B, Wanner C, Lachin JM, et al. Empagliflozin, cardiovascular outcomes, and mortality in type 2 diabetes. N Engl J Med. 2015;373:2117-28.

10. Neal B, Perkovic V, Mahaffey KW, et al. Canagliflozin and cardiovascular and renal events in type 2 diabetes. N Engl J Med. 2017;377:644-57.

11. Seino Y, Sasaki T, Fukatsu A, Ubukata M, Sakai S, Samukawa Y. Efficacy and safety of luseogliflozin as monotherapy in Japanese patients with type 2 diabetes mellitus: a randomized, double-blind, placebo-controlled, phase 3 study. Curr Med Res Opin. 2014;30:1245-55.

12. Nishimura R, Osonoi T, Kanada S, et al. Effects of luseogliflozin, a sodium-glucose co-transporter 2 inhibitor, on 24-h glucose variability assessed by continuous glucose monitoring in Japanese patients with type 2 diabetes mellitus: a randomized, double-blind, placebo-controlled, crossover study. Diabetes Obes Metab. 2015;17:800-4.

13. Seino $\mathrm{Y}, \mathrm{Kaku} \mathrm{K}$, Inagaki N, et al. Fifty-two-week long-term clinical study of luseogliflozin as monotherapy in Japanese patients with type 2 diabetes mellitus inadequately controlled with diet and exercise. Endocr J. 2015;62:593-603.

14. Sakai S, Kaku K, Seino Y, et al. Efficacy and safety of the SGLT2 inhibitor luseogliflozin in Japanese patients with type 2 diabetes mellitus stratified according to baseline body mass index: pooled analysis of data from 52-week phase III trials. Clin Ther. 2016;38:843-62.

15. Qiang S, Nakatsu Y, Seno Y, et al. Treatment with the SGLT2 inhibitor luseogliflozin improves nonalcoholic steatohepatitis in a rodent model with diabetes mellitus. Diabetol Metab Syndr. 2015;7: 104.

16. Kusunoki M, Natsume Y, Sato D, et al. Luseogliflozin, A sodium glucose co-transporter 2 inhibitor, alleviates hepatic impairment in Japanese patients with type 2 diabetes. Drug Res (Stuttg). 2016;66: 603-6.

17. Sumida Y, Murotani K, Saito M, et al. Effect of luseogliflozin on hepatic fat content in type 2 diabetes patients with non-alcoholic fatty liver disease: a prospective, single-arm trial (LEAD trial). Hepatol Res. 2019;49:64-71.

18. Nauck MA, Del Prato S, Meier JJ, et al. Dapagliflozin versus glipizide as add-on therapy in patients with type 2 diabetes who have inadequate glycemic control with metformin: a randomized, 52-week, double-blind, active-controlled noninferiority trial. Diabetes Care. 2011;34:2015-22.

19. Hirose S, Nakajima S, Iwahashi Y, Seo A, Takahashi $\mathrm{T}$, Tamori Y. Impact of the 8-week administration of tofogliflozin for glycemic control and body composition in Japanese patients with type 2 diabetes mellitus. Intern Med. 2016;55:3239-45.

20. Tosaki T, Kamiya H, Himeno T, et al. Sodium-glucose co-transporter 2 inhibitors reduce the abdominal visceral fat area and may influence the renal function in patients with type 2 diabetes. Intern Med. 2017;56:597-604.

21. Sugiyama S, Jinnouchi H, Kurinami N, et al. Dapagliflozin reduces fat mass without affecting muscle mass in type 2 diabetes. J Atheroscler Thromb. 2018;25:467-76.

22. Shibuya T, Fushimi N, Kawai M, et al. Luseogliflozin improves liver fat deposition compared to metformin in type 2 diabetes patients with non-alcoholic fatty liver disease: a prospective randomized controlled pilot study. Diabetes Obes Metab. 2018;20:438-42.

23. Kuchay MS, Krishan S, Mishra SK, et al. Effect of empagliflozin on liver fat in patients with type 2 diabetes and nonalcoholic fatty liver disease: a randomized controlled trial (E-LIFT trial). Diabetes Care. 2018;41:1801-8.

24. Latva-Rasku A, Honka MJ, Kullberg J, et al. The SGLT2 inhibitor dapagliflozin reduces liver fat but does not affect tissue insulin sensitivity: a 
randomized, double-blind, placebo-controlled study with 8-week treatment in type 2 diabetes patients. Diabetes Care. 2019;42:931-7.

25. Aso Y, Kato K, Sakurai S, et al. Impact of dapagliflozin, an SGLT2 inhibitor, on serum levels of soluble dipeptidyl peptidase- 4 in patients with type 2 diabetes and non-alcoholic fatty liver disease. Int J Clin Pract. 2019;73:e13335.

26. Liao $\mathrm{X}$, Wang $\mathrm{X}$, Li $\mathrm{H}$, et al. Sodium-Glucose Cotransporter 2 (SGLT2) Inhibitor increases circulating zinc-alpha2-glycoprotein levels in patients with type 2 diabetes. Sci Rep. 2016;6:32887.

27. Steven S, Oelze M, Hanf A, et al. The SGLT2 inhibitor empagliflozin improves the primary diabetic complications in ZDF rats. Redox Biol. 2017;13: 370-85.

28. Hong AR, Koo BK, Kim SW, Yi KH, Moon MK. Efficacy and safety of sodium-glucose cotransporter-2 inhibitors in Korean patients with type 2 diabetes mellitus in real-world clinical practice. Diabetes Metab J. 2019;43:590-606.

29. Nakatsu Y, Kokubo H, Bumdelger B, et al. The SGLT2 inhibitor luseogliflozin rapidly normalizes aortic mRNA levels of inflammation-related but not lipid-metabolism-related genes and suppresses atherosclerosis in diabetic ApoE $\mathrm{KO}$ mice. Int J Mol Sci. 2017;18:e1704.

30. Jaikumkao K, Pongchaidecha A, Chueakula N, et al. Dapagliflozin, a sodium-glucose co-transporter-2 inhibitor, slows the progression of renal complications through the suppression of renal inflammation, endoplasmic reticulum stress and apoptosis in prediabetic rats. Diabetes Obes Metab. 2018;20:2617-26.

31. Bouchi R, Terashima M, Sasahara Y, et al. Luseogliflozin reduces epicardial fat accumulation in patients with type 2 diabetes: a pilot study. Cardiovasc Diabetol. 2017;16:32.

32. Bonnet F, Scheen AJ. Effects of SGLT2 inhibitors on systemic and tissue low-grade inflammation: the potential contribution to diabetes complications and cardiovascular disease. Diabetes Metab. 2018;44:457-64.

33. Hamaguchi E, Takamura T, Sakurai M, et al. Histological course of nonalcoholic fatty liver disease in Japanese patients: tight glycemic control, rather than weight reduction, ameliorates liver fibrosis. Diabetes Care. 2010;33:284-6.

34. Seko Y, Sumida Y, Tanaka S, et al. Serum alanine aminotransferase predicts the histological course of non-alcoholic steatohepatitis in Japanese patients. Hepatol Res. 2015;45:E53-61.

35. Honda Y, Imajo K, Kato T, et al. The selective SGLT2 inhibitor ipragliflozin has a therapeutic effect on nonalcoholic steatohepatitis in mice. PLoS One. 2016;11:e0146337.

36. Ghosh RK, Ghosh SM, Chawla S, Jasdanwala SA. SGLT2 inhibitors: a new emerging therapeutic class in the treatment of type 2 diabetes mellitus. J Clin Pharmacol. 2012;52:457-63. 